these three micro-organisms is thus very similar in character to that exerted by carbon monoxide.

An atmosphere of carbon dioxide, again, prevented the development of any colonies on the plate-cultures of all three organisms, and only in the case of $B$. pyocyaneus did any appear on transference to air, and then only to the extent of about one-twelfth of the number present on the control air-plates.

In reviewing the record of these experiments made forty-one years ago, it is obvious how greatly the investigation could be extended in various directions. Thus, for example, it would be particularly interesting to ascertain the deportment of sub-cultures made from the colonies appearing on plates which had been exposed to the toxic gases. By pursuing such experiments through a succession of generations it might be possible to arrive at 'strains' of the organisms endowed with the capacity to resist the inhibiting action of a particular gas. Again, differences in behaviour towards a given toxic gas might be made a means of discriminating between otherwise similar organisms, and thus adding yet one more to the already overwhelming number of tests employed in bacteriological diagnosis.

Loch Awe, Argyll.

Percy F. Frankiand.

\section{The First Public Chemical Laboratory in England.}

The statement (Nature, Feb. 19, p. 300) that the chemical laboratory opened in 1828 in University College, London, is the oldest public chemical laboratory in Britain can scarcely be accepted without qualification, in view of the fact that the University of Oxford had built a laboratory fitted for chemical studies so early as 1683, and that this remained in use until superseded in 1848 by Dr. Daubeny's new laboratory at Magdalen College.

The use that was made of it was intermittent, and the modern practice of class teaching necessitating the multiplication of sets of apparatus had not been evolved; but still the possibility for a person to witness an experiment, even if he did not wish to make it himself, was there.

In those early days the University of Oxford appears to have done little for chemical studies beyond the initial provision of the laboratory and of "the Alkanor and Great Reverberatory" furnaces, and larger utensils such as the great alembic, barrel and worm, with which it was equipped. Smaller earthen and glass vessels and chemicals appear to have been the private property of the laboratory assistant for the time being, who was permitted to increase a very meagre salary by selling to experimenters chemical preparations "at easie rates" and by taking payment for the performance of experiments himself.

Notwithstanding the non-existence of any special university teachers of chemistry, useful practical instruction had been given in connexion with courses of public lectures in the laboratory at various times during the eighteenth century. The auditors were largely composed of medical students, with a sprinkling of the more intelligent members of the general public, who, like Princess Anne in 1683, entered the laboratory to see experiments "to their great satisfaction"; and some of the more curiousminded of these would doubtless have sought to try the experiments themselves. Dr. John Freind, described as well skilled in practical chemistry, in 1704 began courses of Prcelectiones Chymicoe in the laboratory, which were partly based on experimental work there, and were printed and reprinted for the next twenty years. Richard Frewin was among those who acted as assistant, and it is not unlikely that there too Dr. John Wall, the inventor of Worcester china, may have learnt his chemical manipulation. George Wingfield has left a written record of the methods of analysis in vogue in 1759, and in 1781 a considerable class of divines is reported to have waded " considerably deep in chemistry." Contemporary notes taken four years later by a pupil of Dr. Martin Wall show the distinctly practical trend of the instruction given, and from 1788 until 1793 the celebrated Dr. Beddoes, later patron to young Humphry Davy, drew to the Ashmolean " the largest classes known in the University since the thirteenth century." It was the period when the work of Sadler, the aeronaut, also helped to popularise chemical experiments in Oxford.

Between 1803 and 1822 the existence of the laboratory made it possible for the first Aldrichian professor of chemistry, Dr. Kidd, to deliver courses of from twenty-six to thirty lectures on the subject of his chair during the winter terms, but owing to the lateness of the hour, 7 P.M., it was unlikely that much work was done in the laboratory by his students. One, at any rate, the poet Shelley, is known to have continued experiments in his untidy rooms in college, while the professor himself worked in the University laboratory at his own researches. Doubtless the dim light of the few candles, or oil lamps, which would have been the only source of illumination then available, as well as the grime of ages on the vaulted ceiling of the laboratory, enhanced that appearance of gloom which has been so often remembered by our visitors during the early years of the nineteenth century, and which was unsuited for the critical operations of analytical chemistry as then practised. But it was not before 1848 that the old laboratory was superseded in Oxford, and the fact remains that for over a hundred and fifty years the Ashmolean had provided England with its first public university laboratory, "the designe of this building being not onlie to advance the studies of true and real philosophy, but also to conduce to the uses of life and the improvement of medicine."

The Old Ashmolean,

R. T. Gunther.

Oxford.

The Spinning Electron in Wave Mechanics.

THE new wave mechanics admits the existence in physical phenomena of a variable quantity that satisfies a special differential equation. According to Schrödinger, this function $\psi$ is such that the product $\psi \bar{\psi}$, where $\bar{\psi}$ is the conjugate complex quantity, is the electrical density. On the contrary Bateman (NATURE, I I 8, 839) has recently shown that, by considering two functions, each of which satisfies the wave equation, it is possible to determine the potentials a and $\phi$ of the electromagnetic field. Starting from Bateman's considerations, de Broglie $(C . R$. I84, 8I) has shown that the values calculated with this theory coincide with those of Maxwell's theory if one admits that the frequency of the fundamental functions be very high and that the considered phenomenon be nearly stationary in relation to this frequency. De Broglie, however, has shown that, given the waveequation

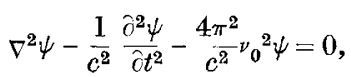

putting in the place of the function $\psi$ the two $\psi_{1}=\frac{A}{r} \cos 2 \pi v_{0} t, \psi_{2}=B \sin 2 \pi v_{0} t$, and introducing the potentials 